DE

M E D I C I N A

T R O P I C A L

$\mathrm{DE}$

SÃO PAULO

JOURNAL OF THE SÃO PAULO INSTITUTE OF TROPICAL MEDICINE

'Universidade Federal de São João DelRei, Campus Centro-Oeste Dona Lindu, Divinópolis, Minas Gerais, Brazil

${ }^{2}$ Universidade de Itaúna, Itaúna, Minas Gerais, Brazil

Correspondence to: Andreia Silva Ferreira Universidade Federal de São João Del-Rei, Campus Centro-Oeste Dona Lindu, R. Sebastião Gonçalves Coelho, 400, CEP 35501-296, Chanadour, Divinópolis, MG, Brazil

E-mail: andreia_rpa@hotmail.com

Received: 10 September 2020

Accepted: 19 January 2021

\section{Biomarkers of severity and chronification in chikungunya fever: a systematic review and meta-analysis}

\author{
Andreia Silva Ferreira ${ }^{(1)}$, Nayara Ragi Baldoni ${ }^{(1,2}$, Clareci Silva Cardoso ${ }^{(1,}$, \\ Claudia Di Lorenzo Oliveira
}

\section{ABSTRACT}

Currently, there are no biomarkers for Chikungunya fever (CHIK) in clinical practice that can accurately predict the severity or chronification of the disease. The aim of this study is to evaluate the existing literature on biomarkers related to the severity and chronification of CHIK. In this sense, a systematic review was conducted based on the PRISMA Statement guideline. Articles that described the association of biomarkers with the evolution of the disease (severity or chronification), published until August $20^{\text {th }} 2019$ were considered eligible. The search was carried out in the PubMed, Scopus, Virtual Health Library (VHL) and Science Direct databases. After searching the databases, 17 articles were added to the review, and after analyzing the articles, several biomarkers were associated with severity, such as increased levels of IL-6, IP-10, IL-1b, MIG, MCP-1, and reduced levels of RANTES and IL-8 or chronification, such as increased levels of IL-6, TNF- $\alpha$, MCP-1, IL-12, INF- $\alpha$, IL-13, INF- $\gamma$, GM-CSF, CRP, IL-1a, IL-15, Factor VII, IP-10, IL-10, IL-4, IL-1RA, IL-8, MIP-1 $\alpha$, MIP-1 $\beta$, ferritin, MIG, ESR, NO, malondialdehyde, and reduced levels of RANTES, ferritin, eotaxin, HGF, IL-27, IL-17A, IL-29, TGF- $\beta$, IL-10, and thiols. IL-6, CRP and TNF- $\alpha$ were included in the meta-analysis to assess the relationship with chronification, although they did not reach statistical significance. It was concluded that several biomarkers showed a relationship with severity and chronification of CHIK; the search for these biomarkers can reveal prognostic factors and important therapeutic targets for the treatment of the disease.

KEYWORDS: Chikungunya virus. Biomarkers. Chronic disease. Severity. Meta-analysis.

\section{INTRODUCTION}

Arboviruses such as dengue, yellow fever, zika, West Nile fever and Chikungunya fever (CHIK) have caused several outbreaks throughout history, and their geographical expansion has caused human and financial losses, becoming today one of the major global public health problems. This expansion can be explained by the increase in the global displacement of people, adaptation of vectors and failure to contain epidemics ${ }^{1}$.

The Chikungunya virus (CHIKV), originally described in Tanzania ${ }^{2}$, had several isolated outbreaks in Africa, and since 2004, a major epidemic started off the coast of Kenya, and spread to numerous islands in the Indian Ocean, India and $\mathrm{Asia}^{3}$. CHIKV maintained a trend towards geographic expansion, reaching the Americas, having its first record on the continent in $2013^{4}$.

CHIK fever is transmitted by mosquitoes of the genus Aedes. The disease manifests with a sudden onset of high fever, cutaneous rashes and severe arthralgia, arthritis, myalgia, headaches and photophobia. Most of the time, it is self-limited, but 
it can evolve to severe conditions ${ }^{5}$. In addition to its potential severity, CHIK fever can progress to chronification. The most common chronic manifestations are musculoskeletal and long-term rheumatic disease, which lead to a worsening of the patients' quality of life ${ }^{6}$.

In the acute phase of the disease, several cytokines are increased such as interleukin 6 (IL-6), tumor necrosis factor alpha (TNF-a), granulocyte macrophage-colony stimulating factor (GM-CSF), macrophage inflammatory protein-1 (MIP-1), and monocyte chemoattraction protein-1 (MCP-1); while in convalescence, lower levels of these cytokines were observed ${ }^{7}$. However, in clinical practice, there is no cytokine or other type of biomarker routinely used to predict the evolution of CHIK fever to severe or chronic conditions.

Considering the expansion of the disease and its potential to evolve to severe cases and chronification, the identification of biomarkers that can predict the outcome of the disease is of great importance. In this context, this systematic review was conducted to identify the existence of biomarkers related to the severity and chronification of CHIKV fever.

\section{MATERIAL AND METHODS}

This systematic review was carried out based on the Preferred Reporting Items for the Systematic Reviews and Meta-Analyzes guideline-The PRISMA Statement ${ }^{8}$, seeking to evaluate the hypothesis that biomarkers can predict the evolution (severity or chronification) of the CHIKV fever. This systematic review was registered on the PROSPERO platform under $\mathrm{N}^{\circ}$ CRD42018094407.

The search for data was carried out in the PubMed, Scopus, Virtual Health Library (VHL), and Science Direct databases including studies published until August $20^{\text {th }} 2019$ without restricting the initial data collection period, since the objective was to recover the maximum available items.

The search strategy used PubMed and Scopus to select the option "advanced search", "title/abstract" to search for the keyword Chikungunya with the Boolean operator AND associating the keywords marker and markers, in turn linked by the operator OR, in the "All Fields" option. In the Science Direct database the same keywords were used, but the Research Articles filter was added, while in the VHL database, the option "title, summary and subject" was used to search for Chikungunya AND marker OR markers.

After selecting articles in each database, duplicate publications were excluded. The search was carried out initially by two researchers (ASF and NRB) reading the abstracts independently, to avoid the presence of bias in the selection and exclusion of studies. After selection, in case of disagreement between the two reviewers, a third and fourth (CLO, CSC) researchers defined the relevance of the abstracts, considering the aims of the investigation. To detect any relevant scientific evidence that may not have been identified by the search strategy, a search for articles was also carried out in the reference list of studies and included in the review.

The articles that met the inclusion criteria were read in full. The collected variables regarding the characteristics of the studies were: name of the first author, year of publication, study reference, study location, language, type of study, sample size, age group, gender, outcome, biomarkers used, study result and laboratory method for analyzing the biomarkers.

To assess the quality of the studies included in this review, the Strengthening Reporting of Observational studies in Epidemiology (STROBE) guideline was used. In this methodology, a scale ranging from 0 to 22 is used, and the higher the score the higher the quality 9

\section{Meta-analysis}

After determining the biomarkers related to chronification and severity, it was decided not to perform a meta-analysis related to severity due to different criteria for defining the term used by each study.

Chronification-related biomarkers, which had data available in more than two studies, were used for metaanalysis, comparing their mean levels and the studies controls.

When relevant data were not found, the authors were contacted by email, and in cases in which there was no success in obtaining the information, the article was excluded from the meta-analysis. After excluding studies with incomplete data, and evaluating the available biomarkers, studies that addressed TNF- $\alpha$, IL- 6 and CRP were included in the meta-analysis. The mean value of the biomarker, its standard deviation, and number of patients in the chronification group and study control group were collected, and meta-analysis was performed to compare the mean difference (MD) of the biomarker between the groups. To create forest plots, we calculated the weighted mean difference (WMD) with $95 \%$ confidence intervals (95\% CIs). Analyzes were performed using the fixed effects model and the random effects model.

The studies included in the meta-analyzes performed the collection of biomarkers at different times during the natural history of the disease (acute or chronic phase) and had different control groups, and to try to control the impact of these, each meta-analysis describes the moment of collection and the control group used. In the case of 
IL-6, it was possible to analyze two variations of the metaanalysis, one grouping studies in which sample collections were carried out in both phases (acute and chronic), and one only with studies performed during the chronic phase. The PCR metanalysis covered chronic phase studies and the TNF-a metanalysis used studies carried out during both phases of the disease.

The $\mathrm{I}^{2}$ index, $\left(\mathrm{I}^{2}\right)$ and the chi-square test was performed to test the heterogeneity among the included studies. A $\mathrm{P}$ value $<0.05$ was considered statistically significant. $\mathrm{I}^{2}>50 \%$ was considered indicative of a high heterogeneity.

One study presented the means, but did not provide standard deviations, thus we performed the imputation of values using the highest value among the studies that addressed the biomarker to obtain a more conservative result.

Additionally, we performed a meta-analysis to calculate the pooled prevalence with $95 \% \mathrm{CI}$ to assess the percentage of chronification in studies that followed-up patients from the acute phase and measured the presence of persistent symptoms. Articles that had a loss of follow-up greater than $50 \%$ of the sample were excluded to reduce the chance of bias. The $\mathrm{R}^{\circledR}$ software with the R Studio ${ }^{\circledR}$ interface were used to perform all the statistical analyzes through the meta and metafor packages, using metaprop and metacont commands.

\section{RESULTS}

The search strategy resulted in the identification of 104 articles in the PUBMED database, 478 in Scopus, 463 in Science Direct and 92 in the VHL, totaling 1,137 manuscripts. In addition, 385 abstracts from the references of selected articles were evaluated to allow them to enter the systematic review. After removing the duplications, 1,281 studies remained, and their titles and abstracts were read resulting in 30 articles selected by two reviewers, independently (ASF \& NRB). The divergences were assessed by a third and fourth reviewers (CLO \& CSC) who excluded five articles because they were considered outside the context of the guiding question (three did not address biomarkers, two were reviews). In total, 25 articles were read in full and after reading them, eight articles were excluded. Among the articles excluded after full reading, two were case reports, two did not address chronification or severity of the disease, and four did not address biomarkers (Figure 1).

Of the $17(100 \%)$ articles included in the review, $13(76 \%)$ addressed chronification ${ }^{10,11-22}$, three articles (18\%) addressed severity ${ }^{23-25}$ and one article (6\%) addressed both themes ${ }^{26}$. Eleven cohort studies ${ }^{10,12-14,16,17,19,21,22,24,26}$, two case-control studies $^{15,18}$ and four sectional studies ${ }^{11,20,23,26}$

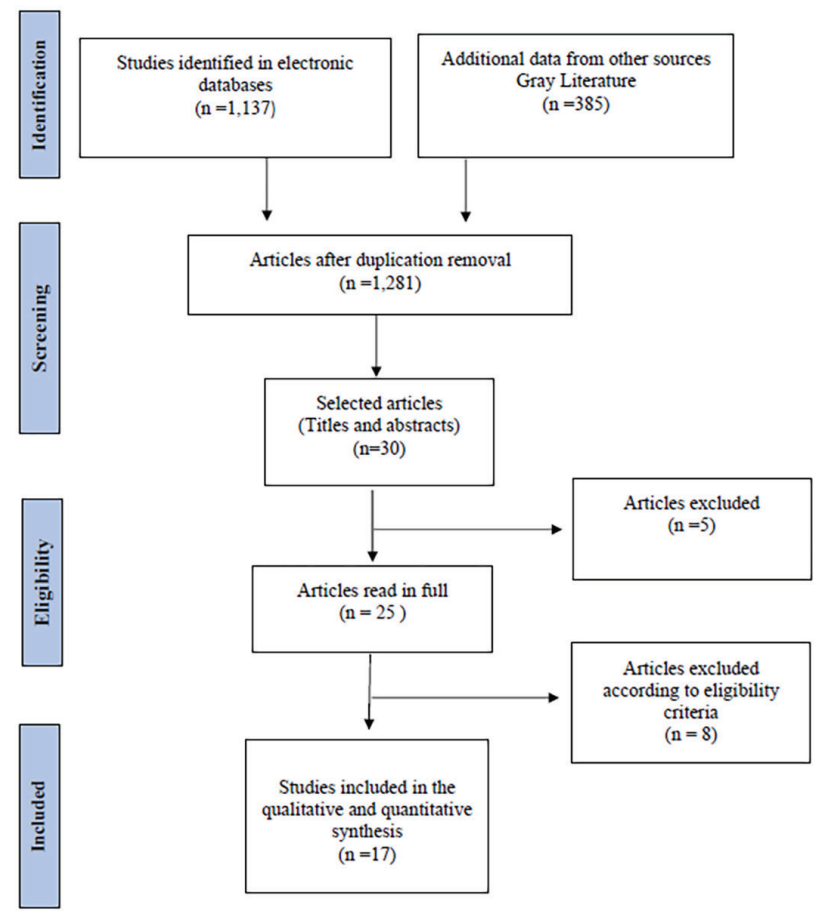

Figure 1 - Flowchart of articles selection based on PRISMA guidelines.

were included. The cohort studies had follow-up times ranging from 1 to 36 months, samples came from India (7 studies) $^{10,11,15,17,21,22,26}$, Singapore (three studies) ${ }^{13,16,23}$, France (two studies) ${ }^{12,14}$, Curacao Island ${ }^{18}$, Mexico $^{19}$, Italy $^{24}$, Thailand ${ }^{25}$ and Brazil ${ }^{20}$ (one from each country). These publications took place between 2009 and 2019, samples were heterogeneous, the age groups ranged from 19 to 90 years. The proportion of patients of each gender also varied, with the percentage of men ranging from $44 \%$ to $100 \%$. (Table 1). The samples size ranged from 10 to 573 participants.

Biomarkers were measured during the acute phase of disease in six studies ${ }^{10,18,21,23,25,26}$, in the subacute/chronic phase in four articles ${ }^{12,13,15,20}$, and was measured in both phases in seven studies ${ }^{11,14,16,17,19,21,24}$. The control group used in each study has also varied, with seven studies using healthy patients ${ }^{10,15,17,20,22-24}$, two used patients with other febrile diseases ${ }^{18,25}$, and eight used patients who had CHIK fever, however they had no symptoms in the chronic phase ${ }^{11-14,16,19,21,26}$ (Table 2 and Table 3).

The criterion for defining severity of CHIKV fever has also varied, and two studies considered as a severe CHIKV fever patient those who had a temperature $>38.5^{\circ} \mathrm{C}$ or heart rate $>100$ beats/minute, or platelet count below $100 \times 10^{9} \mathrm{~g} / \mathrm{L}^{23,25}$. Another study evaluated the severity of arthritis through a visual analog scale ${ }^{26}$ and a survey defined the severity from a questionnaire, created by the authors, which quantified the symptoms ${ }^{24}$. The persistent symptom 
Table 1 - Comparative analysis between articles according to the designs of the studies and characteristics of the evaluated populations $(n=13)$.

\begin{tabular}{|c|c|c|c|c|c|c|c|}
\hline Study & Outcome & Location & Study design & $\begin{array}{l}\text { Time of } \\
\text { follow-up }\end{array}$ & Age range & Mean age & $\begin{array}{l}\text { Gender } \\
(\mathrm{M} / \mathrm{F})\end{array}$ \\
\hline Win et al. ${ }^{13}$ & Chronification & Singapore & Cohort & 1.5 months & $20-79$ & 37 & $75 / 22$ \\
\hline Hoarau et al. ${ }^{14}$ & Chronification & France & Cohort & 12 months & $19-90$ & 60,3 & $19 / 30$ \\
\hline Chopra and Venugopalan ${ }^{15}$ & Chronification & India & Case control & NA & $\mathrm{NI}$ & $\mathrm{NI}$ & $54 / 74^{*}$ \\
\hline Chow et al. ${ }^{16}$ & Chronification & Singapore & Coorte & 2 to 3 months & $23-67$ & 39 & $26 / 4$ \\
\hline Chaaitanya et al. ${ }^{11}$ & Chronification & India & Sectional & NA & $25-55$ & 34.5 & $\mathrm{NI}$ \\
\hline Chopra et al. ${ }^{22}$ & Chronification & India & Cohort & 24 months & $\mathrm{NI}$ & $\mathrm{NI}$ & $226 / 282^{* * *}$ \\
\hline Schilte et al. ${ }^{12}$ & Chronification & France & Cohort & 36 months & $\mathrm{NI}$ & $\mathrm{NI}$ & $73 / 74^{\star * \star}$ \\
\hline Reddy et al. ${ }^{17}$ & Chronification & India & Cohort & 3 months & $21-80$ & 40,6 & $31 / 17$ \\
\hline Venogopalan et al..$^{10}$ & Chronification & India & Cohort & 1 month & $\mathrm{NI}$ & 36 & $44 / 66^{\star \star \star \star *}$ \\
\hline Anfasa et al. ${ }^{18}$ & Chronification & Curaçao Island & Case control & NA & $\mathrm{NI}$ & $\mathrm{NI}$ & $\mathrm{NI}$ \\
\hline Sepúlveda-Delgado et al. ${ }^{19}$ & Chronification & Mexico & Cohort & 12 months & $27-68$ & 48 & $4 / 6$ \\
\hline $\begin{array}{l}\text { Banerjee and } \\
\text { Mukhopadhyay }{ }^{21}\end{array}$ & Chronification & India & Cohort & 0.33 a 1 month & $35-76^{*}$ & $55^{\star}$ & $10 / 15^{*}$ \\
\hline Cavalcanti et al..$^{20}$ & Chronification & Brazil & Sectional & NA & $\mathrm{NI}$ & 55.2 & $8 / 37$ \\
\hline $\mathrm{Ng}$ et $\mathrm{al} .^{23}$ & Severity & Singapore & Sectional & NA & $22-65$ & 35 & $10 / 0$ \\
\hline Kelvin et al. ${ }^{24}$ & Severity & Italy & Cohort & 12 months & $\mathrm{NI}$ & $\mathrm{NI}$ & $\mathrm{NI}$ \\
\hline Lohachanakul et al. ${ }^{25}$ & Severity & Thailand & Sectional & NA & $\mathrm{NI}$ & $34.9^{\star *}$ & $23 / 39^{\star * * \star * *}$ \\
\hline Jain et al. ${ }^{26}$ & $\begin{array}{c}\text { Severity/ } \\
\text { Chronification }\end{array}$ & India & Cohort & 3 months & $17-69$ & 36 & 282/291 \\
\hline
\end{tabular}

${ }^{*}$ Considering only samples with chronic symptoms ${ }^{\star \star}$ Considering only samples with severe symptoms; ${ }^{\star \star \star}$ Considering the followup of 14 months; ${ }^{* \star \star \star}$ Estimated value; ${ }^{* \star \star \star}$ Estimated value considering only samples with chronic symptoms; ${ }^{\star \star \star \star \star \star}$ Considering patient with or without chikungunya; $\mathrm{M}=$ Male; $\mathrm{F}=$ Female; $\mathrm{NI}=$ Not informed; $\mathrm{NA}=$ Not applicable

accompanied by most studies to define subacute or chronic disease was arthralgia, but other symptoms such as arthritis, fatigue, myalgia and low back pain were also assessed.

The biomarkers covered in the studies were leukocyte, lymphocyte and platelet count, liver enzymes dosage, serum creatinine, hemoglobin, haptoglobin, ferritin, fibrinogen, VHS, lactic dehydrogenase (LDH), CRP, IL-1, IL-1a, IL-1RA , IL-1b, IL-2, interleukin -3 (IL-3), IL-4, IL-5, IL-6, interleukin-7 (IL-7), IL-8, IL-10, IL-12, IL-13, IL-15, IL-17, IL-18, interleukin-21 (IL-21), interleukin-22 (IL-22), interleukin-23 (IL-23), interleukin-27 (IL- 27), interleukin-29 (IL-29), IL-1ra, INF- $\alpha$, INF- $\gamma$, TNF- $\alpha$, TNF-b, tumor necrosis factor receptor 2 (TNFR2), MCP-1, IP-10, MIG, CC ligand 5 chemokine regulated by expressed activation and secreted by normal $\mathrm{T}$ cells (RANTES), eotaxin, GM-CSF, hepatocyte growth factor (HGF), transforming growth factor beta (TGF- $\beta$ ), factor VII, C3, anti-cyclic citrullinated peptide (anti-CCP), rheumatoid factor (FR), anti-nuclear antibody (Anti-ANA), Von Willebrand factor, neopterin, matrix metalloproteinases (MMP), tissue inhibitor of metalloproteinases 1 (TIMP-1), MIP-1 $\alpha$, epidermal growth factor (EGF), HGF, FGF-b, VEGF, G-CSF, GM-CSF, MIP-1 $\beta$, vascular cell adhesion molecule 1 (VCAM-1), intercellular adhesion molecule 1 (ICAM-1), vitamin D carrier protein (DBP), alpha-2macroglobulin, alpha-1-antitrypsin (AAT), beta-2- microglobulin (B2M), brain-derived neurotrophic factor (BDNF), stem cell factor antagonist, nitric acid (NO), carbonylated protein, thiols and malondialdehyde (Figure 2).

Chronification was associated with increased levels of IL-6 (6 studies) $)^{10,11,15,16,19,22}$, TNF- $\alpha$ (2 studies $)^{10,15}$, MCP-1 ( 3 studies) ${ }^{10,11,17}, \mathrm{IL}-12^{14}$, INF- $\alpha^{14}, \mathrm{IL}^{1}-13^{15}, \mathrm{INF}-\gamma^{15}$, GM-CSF ${ }^{16}, \mathrm{CRP}^{12}, \mathrm{IL}^{1} \mathrm{a}^{12}, \mathrm{IL}^{1} 15^{12}$, Factor VII ${ }^{12}, \mathrm{IP}^{1} 10^{10}$, IL- $10^{10}$, IL- $4^{10}$, IL-1RA ${ }^{11}$, IL- $8^{11}$, MIP- $1 \alpha^{11}$, MIP- $1 \beta^{11}$, ferritin ${ }^{18}, \mathrm{MIG}^{17}, \mathrm{ESR}^{19}, \mathrm{NO}^{21}$, malondialdehyde ${ }^{21}$ and with reduced levels of RANTES ${ }^{11,17}$, ferritin ${ }^{12}$, eotaxin ${ }^{16}$, HGF $^{16}, \mathrm{IL}^{2}-27^{20}, \mathrm{IL}-17 \mathrm{a}^{20}, \mathrm{IL}-29^{20}$, TGF- $\beta^{21}, \mathrm{IL}-10^{21}$, and thiols $^{21}$ (Figure 2).

Among the increased biomarkers that showed an association with severity were increased levels of IL-6 (2 studies) ${ }^{23,25}$, IP-10 (2 studies) ${ }^{23,24}, \mathrm{IL}^{2} \mathrm{~b}^{23}, \mathrm{MIG}^{24}$, MCP- $1^{25}$ and reduced levels of RANTES ${ }^{23}$, and IL- $8^{25}$ (Figure 2).

Among the articles included in the meta-analysis, the methodology used in each study to measure biomarkers has greatly differed, using Enzyme Linked Immunosorbent Assays (ELISAs) ${ }^{14,15,19}$ or the Multiplex Kit - Luminex ${ }^{16}$ for measuring cytokines, and in the case of measuring CRP one study cited the use of protein electrophoresis ${ }^{12}$.

The studies had scores between 19 and 13 on the STROBE scale; the score of 19 was obtained by three 
Table 2 - Comparative analysis between articles that addressed chronification according to the sample size, chronification index, collection time and characteristics of the control group $(n=13)$.

\begin{tabular}{|c|c|c|c|c|c|}
\hline Study & $\begin{array}{l}\text { Sample } \\
\text { size }\end{array}$ & $\begin{array}{c}\text { Sample with } \\
\text { biomarkers in the } \\
\text { group with persistent } \\
\text { symptoms }\end{array}$ & Control & $\begin{array}{l}\text { Control } \\
\text { sample }\end{array}$ & $\begin{array}{c}\text { Collection time in the } \\
\text { group with persisten } \\
\text { symptoms }\end{array}$ \\
\hline Win et al..$^{13}$ & 97 & 14 & $\begin{array}{l}\text { Cases without } \\
\text { chronification }\end{array}$ & 25 & Chronic phase \\
\hline Hoarau et al. ${ }^{14}$ & 49 & 6 & $\begin{array}{l}\text { Cases without } \\
\text { chronification* }\end{array}$ & 9 & $\begin{array}{c}\text { Acute and chronic } \\
\text { phase }\end{array}$ \\
\hline Chopra and Venugopalan ${ }^{15}$ & 141 & 141 & Healthy patients & 80 & Chronic phase \\
\hline Chow et al. ${ }^{16}$ & 30 & 4 & $\begin{array}{l}\text { Cases without } \\
\text { chronification* }\end{array}$ & 26 & $\begin{array}{c}\text { Acute and Chronic } \\
\text { phase }\end{array}$ \\
\hline Chaaitanya et al..$^{11}$ & 22 & 10 & $\begin{array}{l}\text { Cases without } \\
\text { chronification* }\end{array}$ & 6 & Chronic phase ${ }^{* *}$ \\
\hline Chopra et al. ${ }^{22}$ & 509 & $22^{\star * *}$ & Healthy patients & $\mathrm{NI}$ & $\begin{array}{l}\text { Acute and chronic } \\
\text { phase }\end{array}$ \\
\hline Schilte et al. ${ }^{12}$ & 180 & 20 & $\begin{array}{l}\text { Cases without } \\
\text { chronification }\end{array}$ & 22 & Chronic phase \\
\hline Reddy et al. ${ }^{17}$ & 48 & 4 & Healthy patients & 37 & $\begin{array}{c}\text { Acute and chronic } \\
\text { phase }\end{array}$ \\
\hline Venogopalan et al. ${ }^{10}$ & 509 & 132 & Healthy patients & 80 & Acute phase \\
\hline Anfasa et al. ${ }^{18}$ & 116 & 116 & $\begin{array}{l}\text { Febrile patients } \\
\text { without } \mathrm{CHIK}\end{array}$ & 25 & Acute phase \\
\hline Sepúlveda-Delgado et al. ${ }^{19}$ & 10 & 6 & $\begin{array}{l}\text { Cases without } \\
\text { chronification }\end{array}$ & 4 & $\begin{array}{l}\text { Acute and chronic } \\
\text { phase }\end{array}$ \\
\hline Banerjee and Mukhopadhyay ${ }^{21}$ & 65 & 25 & $\begin{array}{l}\text { Cases without } \\
\text { chronification* }\end{array}$ & 15 & $\begin{array}{c}\text { Acute/subacute } \\
\text { phase }\end{array}$ \\
\hline Cavalcanti et al. ${ }^{20}$ & 45 & $\begin{array}{l}21 \text { (chronic)/ } \\
24 \text { (subacute) }\end{array}$ & Healthy patients & 49 & $\begin{array}{l}\text { Subacute/chronic } \\
\text { phase }\end{array}$ \\
\hline Jain et al. ${ }^{26}$ & 572 & 40 & $\begin{array}{l}\text { Cases without } \\
\text { chronification }\end{array}$ & 27 & Acute phase \\
\hline
\end{tabular}

Table 3 - Comparative analysis between the articles that addressed severity according to the sample size, collection time and characteristics of the control group $(n=4)$.

\begin{tabular}{lccccc}
\hline Study & Sample & $\begin{array}{c}\text { Sample with } \\
\text { severe CHIK }\end{array}$ & Control & $\begin{array}{c}\text { Control } \\
\text { sample }\end{array}$ & Collection time \\
\hline Ng et al. ${ }^{23}$ & 10 & 5 & Healthy patients & 9 & Acute phase \\
Kelvin et al. ${ }^{24}$ & 50 & 14 & Healthy patients & 10 & Acute and chronic phase \\
Lohachanakul et al. ${ }^{25}$ & 62 & 15 & Febrile patients without CHIK & 27 & Acute phase \\
Jain et al. ${ }^{26}$ & 573 & 40 & Cases without severe arthralgia & 27 & Acute phase \\
\hline
\end{tabular}

studies $^{10,12,23}, 18$ points by four other studies ${ }^{14,21,22,24}$ 17 points by five studies ${ }^{11,13,18,19,26}, 16$ points by four studies $^{15,16,20,25}$, and one study obtained 13 points ${ }^{17}$; the average of score was 17 . No article achieved the maximum score.

\section{Meta-analysis}

The assessment of IL-6 levels in the meta-analyzes that grouped studies with patients who evolved to chronicity at any stage of the disease (acute or chronic phase)
$(\mathrm{MD}=-7.65 / 95 \% \mathrm{CI}-34.06-+18,17)$ or only in the chronic phase $(\mathrm{MD}=-2.90 / 95 \% \mathrm{CI}-35.74-+29.94)$, in both cases there was no statistically significant difference in comparison with healthy patients or patients with a previous history of Chikungunya without chronification. Additionally, the evaluation of CRP in the chronic phase $(\mathrm{MD}=-2.70 / 95 \% \mathrm{CI}-16.93-+11.54)$ and the evaluation of TNF-a at any stage of the disease (MD = $-1.91 / 95 \%$ CI $-33.19-+37.01)$ did not show any statistically significant difference when compared to the control group (Figure 3). 


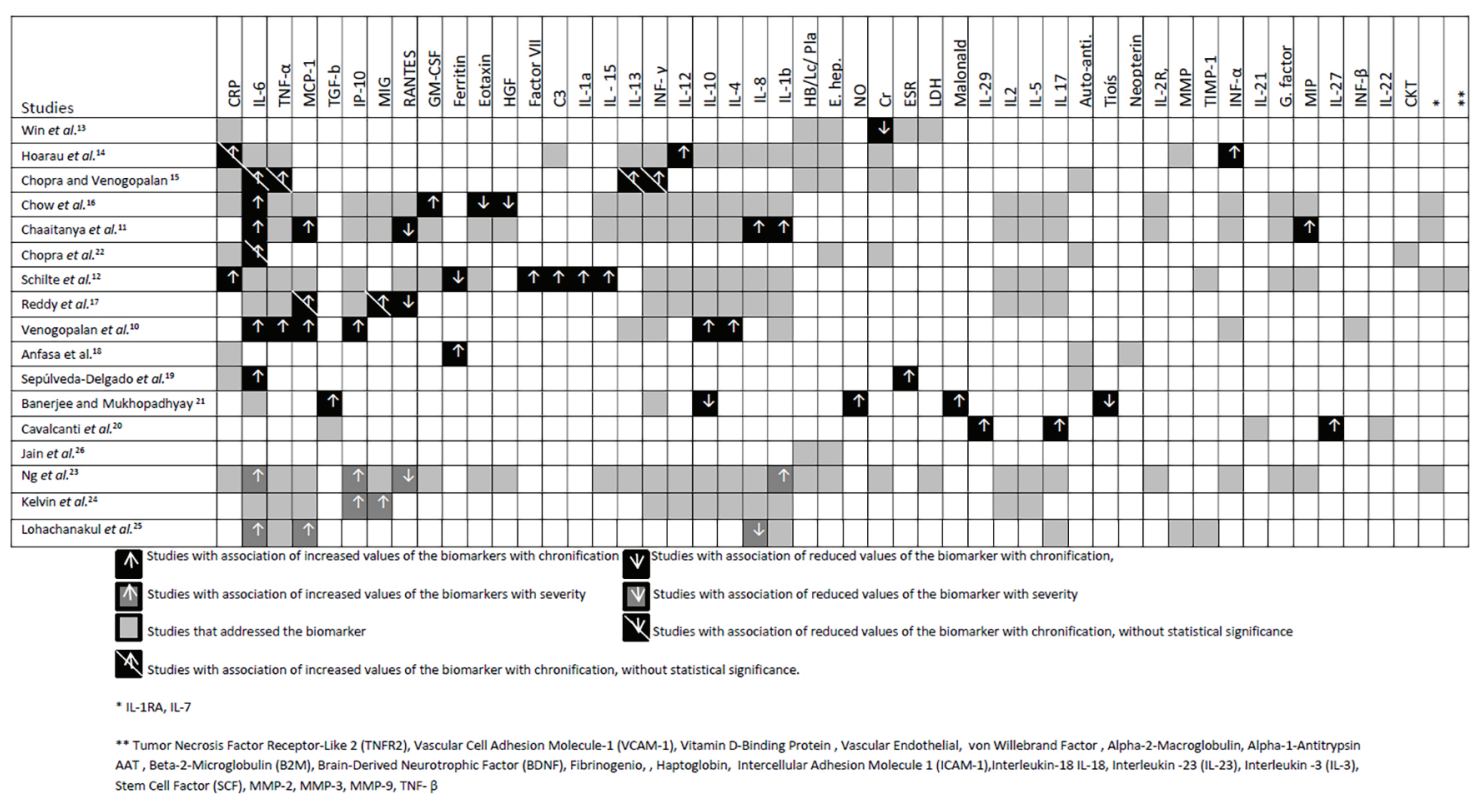

Figure 2 - Chart showing the relationship of the biomarkers addressed with severity or chronification of CHIKV fever according to the authors' assessments.

The proportion of chronification considering only cohort studies that followed patients from the acute phase was $15 \%$ (95\% CI, 0.08-0.26) of chronification among patients who had CHIK fever (Figure 4).

The heterogeneity was statistically significant in the IL-6 meta-analyzes measured in the chronic phase $\left(\mathrm{I}^{2}=72 \%\right.$, $\mathrm{P}=0.03)$, in the evaluation of $\mathrm{CRP}\left(\mathrm{I}^{2}=74 \%, \mathrm{P}=0.02\right)$, and in the assessment of the proportion of the chronic $\left(\mathrm{I}^{2}=87 \%\right.$, $\mathrm{P}=0.01$ ). In addition, the meta-analyzes that evaluated IL-6 in any stage of the disease $\left(\mathrm{I}^{2}=57 \%, \mathrm{P}=0.07\right)$ and in the meta-analysis that evaluated TNF- $\alpha\left(\mathrm{I}^{2}=59 \%, \mathrm{P}=0.09\right)$ showed high $\mathrm{I}^{2}$ values.

\section{DISCUSSION}

Although the meta-analyzes did not show statistically significant relationships, several studies cited biomarkers related to the severity and chronicity of the disease, especially the main biomarkers associated with chronification, being IL-6, TNF- $\alpha$ and MCP-1. These biomarkers may have a potential use as therapeutic targets or prognostic markers in future studies. Another relevant finding is that other biomarkers widely used and easily accessible in practice, such as liver enzymes, renal function and antibodies did not show a relationship with the evolution of the disease. Research on the topic is still scarce so that it is possible that with the increase in the number of studies and the analysis of larger samples, the statistical associations will become more notorious and other associations will be discovered, proven or refuted.

Many studies evaluated the association with ILs and chronification, among them IL-6 was related to chronification by six studies ${ }^{10,11,15,16,19,22}$, Chopra and Venugopalan ${ }^{15}$ evaluated patients with persistent symptoms after one year of illness and observed a marginal increase in IL-6, IFN- $\gamma$, TNF- $\alpha$ and IL-13, a finding that according to the authors, may suggest an ongoing immune inflammatory process. Chopra et al.22 found an eight-fold increment of IL-6 values in patients in comparison with the healthy control group and IL-6 remained elevated during the two-year follow-up period; the researcher argued that the increased levels may be related to viral persistence. Sepulveda-Delgado et al. ${ }^{19}$ observed patients with high levels of IL-6 at the time of diagnosis and ESR developed subacute or chronic disease, but the study conducted by Hoarau et $a l .{ }^{14}$ found no differences, in the acute phase, between patients who progressed or not to chronification in most analyzes, but the levels of circulating IL-12 persisted high for months only in the group that evolved to chronicity. In the study conducted by Chow et al. ${ }^{16}$ patients with persistent arthralgia had increased levels of IL-6 and GM-CSF, while those who recovered had normal levels. In contrast, patients who did not have persistent arthritis had higher levels of HGF, a hormone that facilitates cartilage repair, and eotaxin, a cytokine that indicates the presence of a local anti-inflammatory response.

Banerjeeet and Mukhopadhyay ${ }^{21}$ evaluated inflammatory and oxidative stress parameters in patients with CHIK and observed that high levels of IL- 6 and INF- $\gamma$ are related to the presence of reactive oxygen species (ROS). The study also observed that in the group with persistent arthritis, the levels of malondialdehyde (marker of lipid peroxidation), and nitric oxide (NO) were increased in relation to controls, and the levels of thiols (important agents of enzymatic 
Comparative meta-analysis of the level of IL-6 measured at any stage of the disease between patients who progressed to chronification and patients with a previous history of chlkungunya without chronification or healthy people (pg / ml)

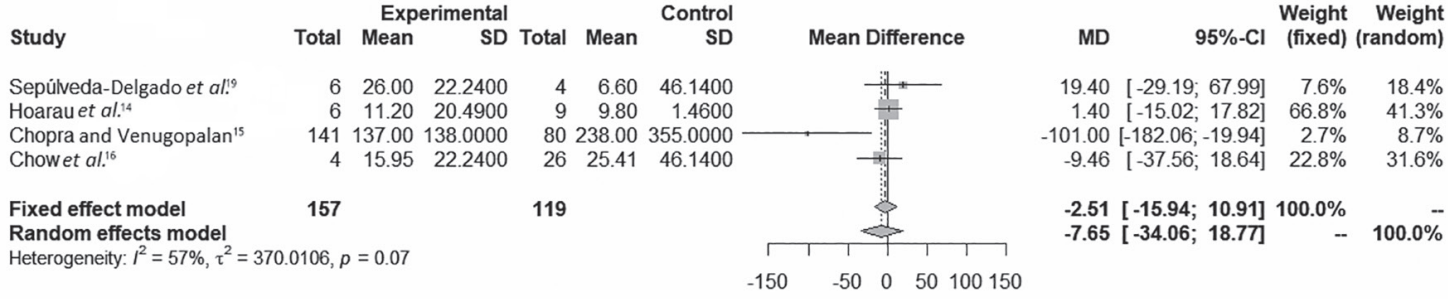

Comparative meta-analysis of the level of IL-6 measured in the chronic phase of the disease between patients who progressed to chronification and patients with a previous history of chikungunya without chronification or healthy people (pg $/ \mathrm{ml}$ ).

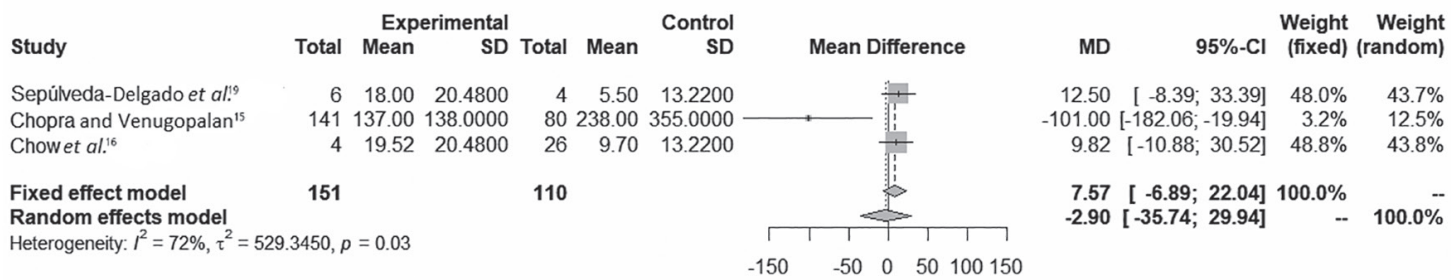

Comparative meta-analysis of the CRP level measured in the chronic phase of the disease among patients who progressed to chronification and with a a previous history of chikungunya without chronification (mg / $\mathrm{dL}$ ).

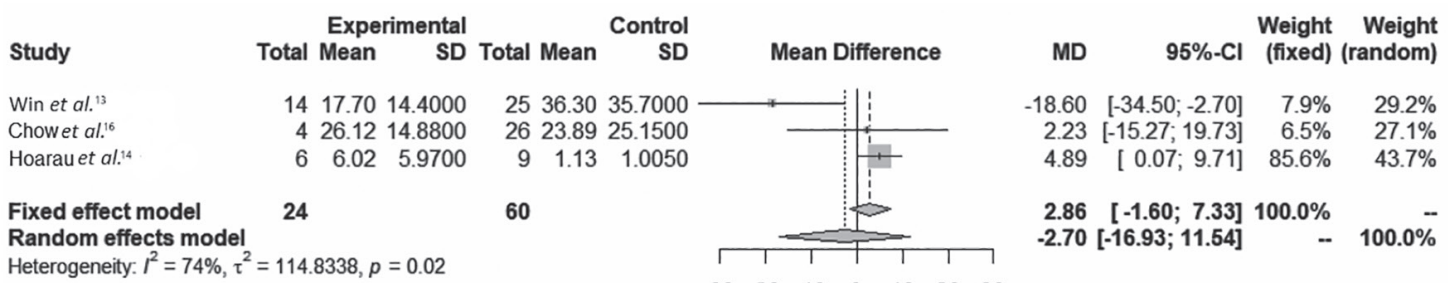

Comparative meta-analysis of the TNF-a level measured at any stage of the disease in patients who progressed to chronification compared to patients with a previous history of chikungunya without chronification or healthy people (pg / ml).

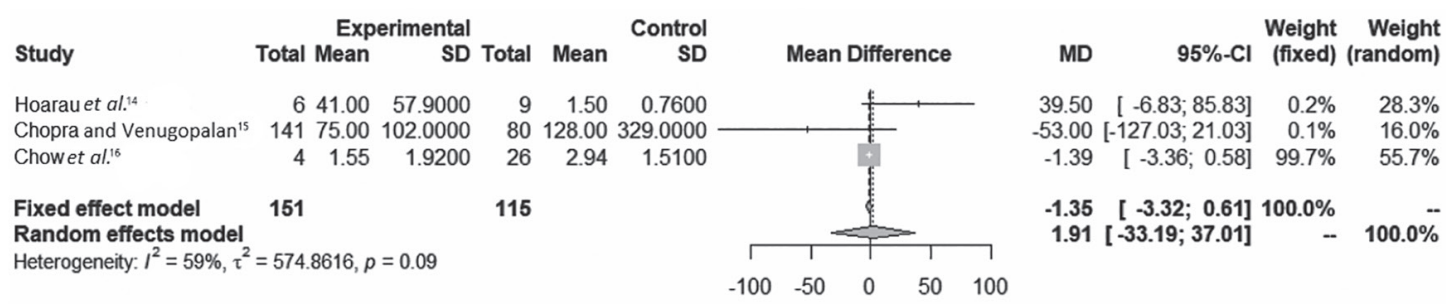

Figure 3 - Comparative meta-analyzes of biomarkers related to chronification of CHIKV fever.

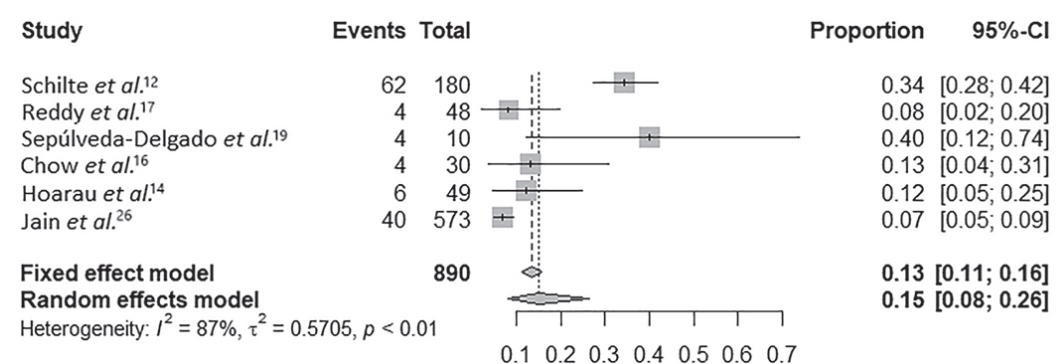

Figure 4 - Meta-analysis on the percentage of chronification among studies used in the systematic review.

activity that can reduce damage caused by the oxidative stress) were reduced in patients with persistent symptoms, showing a tendency towards a greater oxidative stress in this group of patients. In turn, free radicals continuously 
increase the availability of neoantigens, which possibly causes more oxidative damage, including joint damage, and the development of persistent polyarthralgia. Another interesting finding of this research was that the antiinflammatory interleukin IL-10 was significantly reduced in the group with persistent arthralgia. The authors related these reduced levels to the presence of decreased numbers of regulatory T cells in a patient with polyarthralgia secondary to CHIKV, and in turn these cells are IL-10 producers.

The study conducted by Cavalcanti et al. ${ }^{20}$ compared a patient with CHIK fever in the subacute or chronic phase with healthy people and observed increased levels of IL-27, IL-17A, IL-29 and TGF- $\beta$ in sick patients. The levels of IL-27 were significantly higher in patients in the chronic phase, when compared to those in the subacute phase, and the authors propose that IL-27 production can be initiated with antigen presenting cells of monocytic/macrocytic strains, which can act in CHIK fever as reservoirs of the virus or as catalysts in the inflammation process. In addition, IL-27 can also act in a pro-inflammatory way, down regulating the response of regulatory $\mathrm{T}$ cells (Tregs) increasing the Th1 or the Th17 responses in the absence of STAT1. In the study by Chaaitanya et al. ${ }^{11}$, the cytokines IL-1RA, IL-6, IL-8, MIP-1a, MIP-1b and MCP-1, showed elevated levels in patients with chronic arthropathy after CHIKV infection compared with healthy patients. The study has also shown that in the acute phase the levels of IL-1RA and IL- 6 are high, however the level of IL-6 observed in chronic patients was much higher than those of acute phase patients, indicating its strong association with chronic arthritis in patients infected by CHIKV.

MCP-1 has been related to chronification in three studies ${ }^{10,11,17}$. Reddy et al. ${ }^{17}$ reported that patients with persistent arthralgia had high values of IL-1b, IL-4, IL-6, IL-8, IP-10, MCP-1 and MIG during the second week of illness and reduced levels of RANTES. The authors highlighted the chemotactic function of activation and migration of MCP-1 macrophages in the mechanism of induction of joint injury. Venogopalan et al. ${ }^{10}$ observed that cytokines, particularly TNF-a, MCP-1, IL-4, IL-6 and IL-10 showed maximum levels in the extended symptomatic phase and remained high in recovered subjects. Additionally, they found a relationship between the increase in IL-6, IP-10, CRP levels and the persistence of CHIKV musculoskeletal pain. In addition, an early and intense response of IFN- $\alpha$, IFN- $\beta$ and IFN- $\gamma$ was associated with persistent symptoms; the authors associated this response with a failure of the host to eradicate the virus. Chaaitanya et al. ${ }^{11}$, found that the levels of IL-8, MCP-1, MIP-1a and MIP-1b were much higher in patients with chronic arthritis compared to controls, acute patients and patients who had fully recovered. In addition, reduced levels of RANTES have also been observed in chronic patients; MCP-1 acted as a chemotactic factor for monocytes ${ }^{27}$, in turn monocytes and macrophages played an important role in the pathophysiology of arthritis ${ }^{28}$.

TNF- $\alpha$ has also been correlated to chronification in two studies ${ }^{10,15}$. TNF- $\alpha$ is a cytokine mainly induced by macrophages, has a chemotactic effect on several leukocyte populations, is involved in the process of inflammation onset, is an important innate immunity component and has also been related to the pathophysiology of rheumatoid $\operatorname{arthritis}^{29}$.

The immune response and the Th1 or Th2 profile manifested by individuals can also be related to the cytokine profile found. Elevated levels of Th2 cytokines have been associated with prolonged musculoskeletal symptoms (IL-4, IL-6, and IL-10) ${ }^{10}$. Another factor to be considered is that the high viral load was related to the chronicity of the disease; high viral load was associated by Chow et al.${ }^{16}$ with levels of IFN-a, IL-6, IL-12, IL-1RA, IP-10 and MCP-1. Similarly, in the study by Reddy et al. ${ }^{17}$, MCP-1 strongly correlated and IL-6 moderately correlated with high viral load in patients in the acute phase of CHIKV fever.

The association of CRP with chronification has been found in some studies. In the study conducted by Schilte et al. ${ }^{12}$, CRP levels tended to be higher in patients with persistent arthritis. Hoarau et al. ${ }^{14}$ observed that patients who progressed to chronification presented a high level of CRP when compared to healthy controls. Chow et al. ${ }^{16}$ observed high levels of CRP during the acute phase patients who evolved to chronicity, when compared with those of healthy controls and recovered patients. The authors suggested that CRP, due to its low cost, high availability, and because it is related to increased levels of viral load, could help in the early identification and monitoring of patients with CHIK at risk of serious disease.

Other factors have been considered in some studies, such as creatinine levels and ferritin. Win et al. ${ }^{13}$ observed that creatinine was significantly lower in patients with persistent arthralgia, but it was more prominent in women, however women had lower levels of creatinine, which could explain this result. In the same study, there was a tendency of thrombocytopenia and high levels of liver enzymes associated with slow recovery from joint pain. Regarding ferritin, Anfasa et al. ${ }^{18}$ found an association between increased serum ferritin in the acute phase of the disease and prolonged arthralgia. In addition, they have also associated increased levels of ferritin with increased neopterin, which is a specific marker of macrophage activation. Increased levels of ferritin and neopterin are markers of macrophage activation, and ferritin synthesis 
can be triggered by cytokines and iron; the study showed that increased ferritin was associated with the absence of viremia. Macrophages and hepatocytes are producers and secretors of ferritin, in turn the production of this compound can be activated during viral replication. Conversely, Schilte et al. ${ }^{12}$ found a relationship between low levels of ferritin and the chronification of the disease. The divergences in the findings do not allow a conclusion to be drawn, and further studies are needed to assess the true impact of ferritin in CHIKV fever.

The profile of biomarkers of patients who progressed with persistent symptoms changed when assessed at the beginning of the disease onset compared to the period of prolonged illness. This finding is found in the study of Reddy et $a l .{ }^{17}$, who followed-up four patients with persistent symptoms up to 12 months after the disease onset. The authors observed that although several markers were altered during the acute phase (IL-1b, IL-4, IL-6, IL-8, IP-10, MCP-1, MIG, and RANTES), most returned to values close to normal in the follow-up. However, MCP-1 and MIG were persistently elevated in patients during the chronic phase, while RANTES levels remained low during the acute phase with a tendency to decline even further during the chronic phase.

Schilte et al. ${ }^{12}$ evaluated 20 patients with chronic arthralgia 36 months after the onset of the disease, who did not have lymphopenia, and the plasma protein levels measured by electrophoresis remained within the normal range, but the CRP levels were significantly elevated in the patients with arthralgia. In addition, the values of factor VII, C3, IL-1a and IL-15 were also high in this phase.

In the present review, around $15 \%$ of patients evolved to chronification in the studies evaluated, although the values obtained cannot be extrapolated to other contexts, since the meta-analysis was not designed to specifically evaluate the chronification rate. A similar value is cited in the literature that points to a percentage of around $14 \%$ of evolution to chronic arthritis, a symptom evaluated by most studies in this review. On the other hand, considering other types of manifestations of the disease, the persistence rate of nonspecific arthralgia and musculoskeletal symptoms can reach $25 \%{ }^{30}$.

In the acute phase, some markers were related to the severity of the disease. However, it is important to highlight that the severity was classified in a very heterogeneous way in the evaluated studies. $\mathrm{Ng}$ et al..$^{23}$ classified as severe, patients with temperatures equal to or above $38.5^{\circ} \mathrm{C}$, a heart rate $>100$ beats/minute or platelets lower than $100 \times 10^{9} \mathrm{~g} / \mathrm{L}$ and found a relationship between severity and increased levels of IL-1b and IL-6 or reduced levels of RANTES. When analyzing these findings, the author highlighted that IL-1b and IL- 6 are pyrogens and may be responsible for the high fever. In addition, IL-1b can mediate the development of arthralgia, since these cytokines are involved in the pathogenesis of several other forms of arthritis, such as rheumatoid arthritis. In turn, RANTES is a T-cell chemokine and its main reservoir in peripheral blood are platelets, which are typically reduced in CHIK infection. CRP was not associated with severity. The study by $\mathrm{Ng}$ et al. ${ }^{23}$ evaluated patients in the acute phase and found high CRP values in 60\% of the patients, but did not identify a relationship between the acute phase marker and the severity of the disease.

Lohachanakul et al. ${ }^{25}$ has also used the same previously mentioned criteria, and observed a relationship between high concentrations of IL- 6 and MCP-1, in addition to low IL-8 titers with the severity of the disease. The authors point out that IL- 6 is a potent pyrogen and could mediate joint pain by sensitizing the nociceptors in the region, in addition to being able to participate in the pain mechanism in local neurons, associated with the inflammatory process. The authors have also highlighted the role of MCP-1 in the induction of monocytes/macrophages, which are involved in the pathophysiology of arthritis, in addition to mentioning that low levels of IL-8 are consistent with severity of disease, since IL-8 fights viral transcription and promotes viral clearance.

Kelvin et al. ${ }^{24}$ classified the severity of patients qualitatively using a questionnaire on the severity of symptoms. They observed that patients with high levels of MIG, IP-10 and IgG had more severe symptoms, these levels had a tendency to decrease, but still remained elevated after six months. The high levels of MIG, IP-10 and IL-6 present in the acute phase are related, by the authors, to a possible consequence of the activation of the $T$ cell adaptive immunity. In addition, they pointed out that MIG and IP-10 are chemokines induced by IFN- $\gamma$ and they participate in the regulation of monocyte/macrophage migration, memory $\mathrm{T}$ cells and NK cells, and can contribute to the persistent immune activation in CHIK disease, leading to chronic symptoms.

Severity-related biomarkers are also studied in other arboviruses, such as dengue, a disease with a clinical presentation similar to CHIK fever. The dengue virus (DENV)-infected endothelial cells produce chemokine and cytokine responses that activate or recruit immune cells to the endothelium. The most serious form of the disease, the hemorrhagic dengue fever, present a 'cytokine storm', with high levels of circulating cytokines and chemokines. Some studies have shown that elevated levels of IL-6, IL-10, IFN- $\gamma$, MIF and CCL-4 could be used as potential predictors of severity in dengue. Furthermore, increased levels of IFN- $\gamma$, TNF- $\alpha$, IL-1 $\beta$, IL-4, IL-6, IL-7, IL-8, IL10, 
IL-13, IL-15, IL-17, IL- 18, MIF, CCL2, CCL4, CCL5 and IP-10 have been reported in patients with hemorrhagic dengue fever, when compared to dengue fever. Thus, factors associated with the endothelium, such as the increase in Ang-2, vWF, sVCAM, VEGF and VEGFR1 levels, and decrease in Ang-1, ADAMTS-13 and VEGFRII levels might serve as biomarkers of severity in dengue. Liver injury is associated with severe dengue disease with the increase in serum aspartate aminotransferase (AST), alanine aminotransferase (ALT), gamma-glutamyl transpeptidase, alkaline phosphatase and serum albumin concentrations ${ }^{31}$. There are also reports that CRP levels in the early stages following the onset of fever may be prognostic for severe dengue. Platelets are also mentioned in some studies, and platelet associated IgM was suggested to be a predictor of hemorrhagic dengue fever. Platelets bind to DENV which activates them, this might indicate a coagulation response involving DENV immune complexes formation ${ }^{32}$. In comparison with the findings in CHIK fever, some interleukins are mentioned in both cases (IL-6 IP-10, IL-1 $\beta$ ), which may correspond to an initial inflammatory phase present in the severe phase of both diseases. The studies included in this review do not report on the inclusion of biomarkers related to the endothelium to assess their relationship with severe forms of CHIK fever; in addition, other increased biomarkers such as liver enzymes, CRP and the number of platelets were not mentioned in the studies as factors related to the severity of CHIKV, however few studies have been carried out on the subject, making possible to find new biomarkers and parallels with other arboviruses according to the advance of research in the area.

It is important to mention that the studies are divergent in many key methodological aspects such as: sample size, length of follow-up time, stage of the disease at the time of blood collection, sample sources and different control groups. In addition, the cohorts show significant follow-up losses, and samples for the study of biomarkers were taken from a very limited number of patients, either because the total sample of the studies was already limited, or because only a small number of patients underwent more in-depth laboratory analysis. The methods of analysis for each biomarker has also differed, although the studies have shown a good correlation between the ELISA method and the Multiplex Cytokine Measurement Kits, often finding very close values ${ }^{33}$, a fact that reduces the chances of bias, since these were the main methods cited by the studies.

\section{CONCLUSION}

Our study has some limitations. Firstly, we can point out the fact that not all studies were included in the meta- analysis due to the lack of available data. Despite the attempts to obtain data from the authors, the feedback obtained was low, probably because some articles were published a long time ago or because the authors have changed their electronic mail addresses and information has not been updated, because many of the unexpected or undesirable results were not published and bias of publication is a concern in all systematic reviews.

CHIK fever is a public health problem with the potential for serious complications. To date, there are no widespread exams in the clinical practice of prognostic evaluation, in addition, the study of the pathophysiology of the disease is fundamental for the development of new therapeutic targets. Several biomarkers are cited in the literature related to severity (increased levels of IL-6, IP-10, IL-1b, MIG, MCP-1 as well as reduced levels of RANTES and IL-8) or chronification (increased levels of IL-6, TNF- $\alpha$, MCP-1, IL-12, INF- $\alpha^{14}$, IL-13, INF- $\gamma$, GM-CSF, CRP, IL-1a, IL-15, factor VII, IP-10, IL-10, IL4, IL-1RA, IL-8, MIP-1 $\alpha$, MIP-1 $\beta$, ferritin, MIG, ESR, NO, malondialdehyde, and reduced levels of RANTES, ferritin, eotaxin, HGF, IL-27, IL-17A, IL-29, TGF- $\beta$, IL-10 and thiols). The search for these biomarkers can reveal prognostic factors and important therapeutic targets for the treatment of the disease. However, the evaluated studies have shown differences and weaknesses that make it difficult to compare results. In this sense, more standardized studies with larger samples are needed to consolidate these findings.

\section{REFERENCES}

1. Gould E, Pettersson J, Higgs S, Charrel R, Lamballeriea X. Emerging arboviruses: why today? One Health. 2017;4:1-13.

2. Lumsden WH. An epidemic of virus disease in Southern Province Tanganyika Territory, in 1952-53. II. General description and epidemiology. Trans R Soc Trop Med Hyg. 1955;49:33-57.

3. Staples JE, Breiman RF, Powers AM. Chikungunya fever: an epidemiological review of a re-emerging infectious disease. Clin Infect Dis. 2009;49:942-8.

4. Faria NR, Lourenco J, Cerqueira EM, Lima MM, Pybus O, Alcantara LC. Epidemiology of Chikungunya virus in Bahia, Brazil, 2014-2015. PLoS Curr. 2016;1:1-8.

5. Sharma SK, Jain S. Chikungunya: a rheumatologist's perspective. Int J Rheum Dis. 2018;21:584-601.

6. Brito CA. Alert: severe cases and deaths associated with Chikungunya in Brazil. Rev Soc Bras Med Trop. 2017;50:5859.

7. Chirathaworn C, Poovorawan Y, Lertmaharit S, Wuttirattanakowit N. Cytokine levels in patients with Chikungunyavirus infection. Asian Pac J Trop Med. 2013;6:631-4. 
8. Moher D, Liberati A, Tetzlaff J, Altman DG. Preferred reporting items for systematic reviews and meta-analyses: the PRISMA Statement. Ann Intern Med. 2009;151:264-9.

9. von Elm E, Altman DG, Egger M, Pocock SJ, Gotzsche PC, Vandenbroucke JP. The strengthening the reporting of observational studies in epidemiology (STROBE) statement: guidelines for reporting observational studies. Int J Surg. 2014;12:1495-9.

10. Venugopalan A, Ghorpade RP, Chopra A. Cytokines in acute Chikungunya. PLoS One. 2014;9:e111305.

11. Chaaitanya IK, Muruganandam N, Sundaram SG, Kawalekar O, Sugunan AP, Manimunda SP, et al. Role of proinflammatory cytokines and chemokines in chronic arthropathy in Chikungunya infection. Viral Immunol. 2011;24:265-71.

12. Schilte C, Staikovsky F, Couderc T, Madec Y, Carpentier F, Kassab S, et al. Chikungunya virus-associated long-term arthralgia: a 36-month prospective longitudinal study. PLoS One. 2013;7:e2137.

13. Win MK, Chow A, Dimatatac F, Go CJ, Leo YS. Chikungunya fever in Singapore: acute clinical and laboratory features, and factors associated with persistent arthralgia. J Clin Virol. 2010;49:111-4.

14. Hoarau JJ, Bandjee MC, Trotot PK, Das T, Li-Pat-Yuen G, Dassa $B$, et al. Persistent chronic inflammation and infection by Chikungunya arthritogenic response alphavirus in spite of a robust host immune response. J Immunol. 2010;184:5914-27.

15. Chopra A, Venugopalan A. Persistent rheumatic musculoskeletal pain and disorders at one year post-Chikungunya epidemic in south Maharashtra: a rural community based observational study with special focus on naive persistent rheumatic musculoskeletal cases and selected cytokine expression. Indian J Rheumatol. 2011;6 Suppl:5-11.

16. Chow A, Her Z, Ong EK, Chen J, Dimatatac F, Kwek DJ, et al. Persistent arthralgia induced by Chikungunya virus infection is associated with interleukin-6 and granulocyte macrophage colony-stimulating factor. J Infect Dis. 2011;203:149-57.

17. Reddy V, Mani RS, Desai A, Vasanthapuram R. Correlation of plasma viral loads and presence of Chikungunya IgM antibodies with cytokine/chemokine levels during acute Chikungunya virus infection. J Med Virol. 2014;86:1393-401.

18. Anfasa F, Provacia L, GeurtsvanKessel C, Wever R, Gerstenbluth I, Osterhaus AD, et al. Hyperferritinemia is a potential marker of chronic Chikungunya: a retrospective study on the Island of Curacao during the 2014-2015 outbreak. J Clin Virol. 2017;86:31-8.

19. Sepúlveda-Delgado J, Vera-Lastra OL, Trujillo K, CansecoÁvila LM, Sánchez-González RA, Gómez-Cruz O, et al. Inflammatory biomarkers, disease activity index, and selfreported disability may be predictors of chronic arthritis after Chikungunya infection: brief report. Clin Rheumatol. 2017;36:695-9.
20. Cavalcanti NG, Vilar KM, Duarte AL, Rêgo MJ, Pereira MC, Pitta IR, et al. IL-27 in patients with Chikungunya fever: a possible chronicity biomarker? Acta Trop. 2019;196:48-51.

21. Banerjee N, Mukhopadhyay S. Oxidative damage markers and inflammatory cytokines are altered in patients suffering with post-chikungunya persisting polyarthralgia. Free Radic Res. 2018;52:887-95.

22. Chopra A, Anuradha V, Ghorpade R, Saluja M. Acute Chikungunya and persistent musculoskeletal pain following the 2006 Indian epidemic: a 2-year prospective rural community study. Epidemiol Infect. 2012;140:842-50.

23. Ng LF, Chow A, Sun YJ, Kwek DJ, Lim PL, Dimatatac F, et al. IL-1b, IL-6, and RANTES as biomarkers of Chikungunya severity. PLoS One. 2009;4:e4261.

24. Kelvin AA, Banner D, Silvi G, Moro ML, Spataro N, Gaibani $\mathrm{P}$, et al. Inflammatory cytokine expression is associated with Chikungunya virus resolution and symptom severity. PLoS Negl Trop Dis. 2011;5:e1279.

25. Lohachanakul J, Phuklia W, Thannagith M, Thonsakulprasert T, Ubol S. High concentrations of circulating interleukin-6 and monocyte chemotactic protein- 1 with low concentrations of interleukin-8 were associated with severe Chikungunya fever during the 2009-2010 outbreak in Thailand. Microbiol Immunol. 2012;56:134-8.

26. Jain J, Nayak K, Tanwar N, Gaind R, Gupta B, Shastri JS, et al. Clinical, serological, and virological analysis of 572 Chikungunya patients from 2010 to 2013 in India. Clin Infect Dis. 2017;65:133-40.

27. Palomino DC, Marti LC. Chemokines and immunity. Einstein (Sao Paulo). 2015;13:469-73.

28. Tanabe IS, Tanabe EL, Santos EC, Martins WV, Araújo IM, Cavalcante $\mathrm{MC}$, et al. Cellular and molecular immune response to Chikungunya virus infection. Front Cell Infect Microbiol. 2018;8:345

29. Bradley JR.TNF-mediated inflammatory disease. J Pathol. 2008;214:149-60.

30. Rodríguez-Morales AJ, Cardona-Ospina JA, Urbano-Garzon SF, Hurtado-Zapata JS. Prevalence of post-Chikungunya infection chronic inflammatory arthritis: a systematic review and metaanalysis. Arthritis Care Res (Hoboken). 2016;68:1849-58

31. John DV, Lin YS, Perng GC. Biomarkers of severe dengue disease: a review. J Biomed Sci. 2015;22:83.

32. Rathore AP, Faroukv FS, St. John AL. Risk factors and biomarkers of severe dengue. Curr Opin Virol. 2020;43:1-8

33. Richens JL, Urbanowicz RA, Metcalf R, Corne J, O'Shea P, Fairclough L. Quantitative validation and comparison of multiplex cytokine kits. J Biomol Screen. 2010;15:562-8. 